\title{
IDENTIFICAÇÃo DE BIÓTIPOS DE AZEVÉM (Lolium multiflorum) Resistentes aO Herbicida Glyphosate em Pomares de MaÇã ${ }^{1}$
}

\author{
Identification of Glyphosate-Resistant Ryegrass (Lolium multiflorum) Biotypes in \\ Apple Orchards
}

VARGAS, L. ${ }^{2}$, ROMAN, E.S. ${ }^{3}$, RIZZARDI, M.A. ${ }^{4}$ e SILVA, V.C. ${ }^{5}$

\begin{abstract}
RESUMO - O glyphosate é um herbicida de amplo espectro utilizado há mais de 15 anos em pomares de maçã na região de Vacaria-RS, para manejo da vegetação nas linhas da cultura. São realizadas, em geral, três a quatro aplicações por ciclo e a dose normalmente utilizada é de 720 a 1.080 g e.a. ha ${ }^{-1}$ de glyphosate ( 2 a 3 L ha ${ }^{-1}$ do produto comercial). O azevém (Lolium multiflorum) é uma planta daninha comum em pomares e, tradicionalmente, sensivel ao glyphosate. Entretanto, nos últimos anos a ocorrência de plantas de azevém que, após receberem o tratamento com glyphosate, não manifestam sintomas significativos de toxicidade sugere que elas adquiriram resistência ao produto. Assim, com o objetivo de avaliar a resposta de uma população de plantas de azevém ao glyphosate, foram realizados três experimentos: um em campo e dois em casa de vegetação. No experimento em campo os tratamentos avaliados constaram de doses crescentes de glyphosate $(0,360,720,1.440$, $2.880,5.760$ e 11.520 g e.a. ha ${ }^{-1}$ ), e os herbicidas paraquat, glufosinate, haloxyfop e diclofop foram empregados como produtos-padrão, aplicados em dois estádios vegetativos do azevém. No experimento em casa de vegetação, os tratamentos constaram de doses crescentes de glyphosate $\left(0,360,720,1.440,2.880\right.$ e $\left.5.760 \mathrm{~g} \mathrm{e.a.} \mathrm{ha}^{-1}\right)$ mais os herbicidas testemunhas, aplicados sobre plantas do biótipo considerado resistente e de um sensível. No segundo experimento realizado em casa de vegetação foram avaliados tratamentos contendo glyphosate $\left(720,1.440,2.880,720+720\right.$ e $720+1.440$ g e.a. ha- $\left.{ }^{-1}\right)$, em aplicações únicas e seqüenciais, mais os herbicidas paraquat, glufosinate, haloxyfop, clethodim, sethoxydim, diclofop, fenoxaprop, fluazifop, paraquat + diuron, atrazine + simazine, trifluralin e metolachlor. A toxicidade dos tratamentos herbicidas foi avaliada aos 15, 30 e 45 DAT (dias após tratamento). Os resultados obtidos nos experimentos em campo e em casa de vegetação, de forma geral, evidenciam que o biótipo sensivel é facilmente controlado com o herbicida glyphosate e pelos demais herbicidas pós-emergentes avaliados, independentemente do estádio vegetativo. Demonstram, ainda, que o biótipo resistente apresenta-se, igualmente ao biótipo sensivel, altamente suscetivel aos herbicidas com mecanismo de ação distinto daquele do glyphosate. No entanto, o biótipo resistente apresenta baixa resposta ao herbicida glyphosate, mesmo se este for empregado em altas doses, evidenciando ter adquirido resistência a esse produto.
\end{abstract}

Palavras-chave: resistência, inibidores da EPSPs, fruticultura.

ABSTRACT - Glyphosate is a wide spectrum herbicide used for over 15 years in apple orchards in Vacaria-RS for weed control in rows of trees. Usually, 3 to 4 applications per year are made at a rate of 720 to $1080 \mathrm{~g}$ a.e. glyphosate ha $\mathrm{h}^{-1}$ (2 to $3 \mathrm{~L} \mathrm{ha}^{-1}$ of commercial product). Ryegrass (Lolium multiflorum) is a common weed in orchards and traditionally sensitive to glyphosate. However, in the last years, some ryegrass plants have not been found to show significant toxicity symptoms after treatment with glyphosate, suggesting that they acquired resistance to this product. To evaluate

Recebido para publicação em 19.2.2004 e na forma revisada em 17.12.2004.

Eng.-Agrônomo, D.S., Pesquisador da Embrapa Uva e Vinho, Caixa Postal 1513, 95200-000 Vacaria-RS, $<$ leandro@cnpuv.embrapa.br>. ${ }^{3}$ Eng.-Agrônomo, Ph.D., Pesquisador da Embrapa Trigo, Caixa Postal 451, 99001-970 Passo Fundo-RS, <eroman@cnpt.embrapa.br>. ${ }^{4}$ Eng.-Agrônomo, Doutor, Professor da Faculdade de Agronomia e Medicina Veterinária da Universidade de Passo Fundo - UPF, 99001-970 Passo Fundo-RS, <rizzardi@upf.tche.br>. ${ }^{5}$ Biólogo, Laboratorista da Embrapa Uva e Vinho. 
the response of a ryegrass plant population to glyphosate, one field and two greenhouse experiments were carried out. The field experiment treatments had increasing rates of glyphosate $10 ; 360 ; 720$; 1,440; 2,880; 5,760 and $11,520 \mathrm{~g}$ a.e. $\left.\mathrm{ha}^{-1}\right)$, in addition to the herbicides paraquat, glufosinateammonium, haloxyfop and diclofop-methyl as standards, sprayed at two different vegetative growth stages of ryegrass. The greenhouse experiments had increasing rates of glyphosate 10; 360; 720; 1,440; 2,880 and 5,760 g a.e. $\mathrm{ha}^{-1}$ ) plus the above listed check herbicides sprayed on biotypes considered resistant and on plants of one susceptible biotype. In the second greenhouse experiment, glyphosate rates (720; 1,440; 2,880; $720+720$ and $720+1,440 \mathrm{~g}$ a.e. ha $\mathrm{h}^{-1}$ ) were sprayed in single and sequential applications, in addition to the herbicides paraquat, glufosinate-ammonium, haloxyfop, clethodim, sethoxydim, diclofop-methyl, fenoxaprop, fluazifop, paraquat + diuron, atrazine + simazine, trifluralin, and metolachlor. Toxicicity to the herbicides was assessed at 15, 30 and 45 DAT (days after treatment). Overall, the field and greenhouse experiment results showed that the susceptible biotype is easily controlled by glyphosate and by the other postemergence herbicides tested, independent of the vegetative growth stage. In addition, the results showed that the resistant biotype, similarly to the susceptible biotype, is highly sensitive to herbicides with mode of action differing from that of glyphosate. However, the resistant biotype presents low response to glyphosate, even if used in high rates, showing that some biotypes acquired resistance to this product.

Key words: resistance, EPSP synthase-inhibitors, fruitculture.

\section{INTRODUÇÃO}

O glyphosate é um herbicida de amplo espectro utilizado há mais de 15 anos em pomares de maçã, na região de Vacaria-RS, para manejo da vegetação nas linhas da cultura. O uso desse herbicida no controle de plantas daninhas em pomares é prática comum na região de Vacaria-RS; o custo relativamente baixo, a alta eficiência sobre diferentes espécies, independentemente do estádio vegetativo nas aplicações, e a facilidade de aplicação são os principais motivos da preferência dos produtores por essa forma de controle e, especificamente, pelo glyphosate. Em pomares, de forma geral, são realizadas de três a quatro aplicações do produto por ciclo, e a dose normalmente utilizada está entre 720 e 1.080 g e.a. ha ${ }^{-1}$ de glyphosate $\left(2\right.$ a $3 \mathrm{~L} \mathrm{ha}^{-1}$ do produto comercial).

O azevém (Lolium multiflorum) é uma espécie anual de inverno que se caracteriza como a planta daninha mais comum em pomares de maçã na região de Vacaria-RS, sendo tipicamente sensivel ao glyphosate. Entretanto, nos últimos anos, os produtores vêm observando redução na resposta do azevém a tratamentos com esse herbicida. A ocorrência de plantas de azevém que, após receber o tratamento, não manifestam sintomas significativos de toxicidade tornou-se comum em pomares de maçãs, sugerindo que estas podem ter adquirido resistência ao herbicida.

A resistência é a capacidade adquirida de uma planta de sobreviver a determinados tratamentos herbicidas que, em condições normais, controlam os integrantes da população. A resistência pode ocorrer naturalmente (seleção) ou ser induzida com uso da biotecnologia (Weed, 1999). O uso repetido de uma molécula herbicida pode selecionar biótipos resistentes de plantas daninhas preexistentes na população, levando ao aumento do seu número (Powles \& Holtum, 1994). Em geral, espécies ou biótipos de uma espécie que melhor se adaptam a uma determinada prática são selecionados e multiplicam-se rapidamente (Holt \& Lebaron, 1990). Evidências sugerem que o aparecimento de resistência a um herbicida em uma população de plantas seja devido à seleção de genótipos resistentes preexistentes, que, devido à pressão de seleção exercida por repetidas aplicações de um mesmo herbicida, encontram condições para multiplicação (Betts et al., 1992).

O número de espécies resistentes ao glyphosate identificadas está aumentando; atualmente são reconhecidas seis espécies resistentes em 20 diferentes regiões (Weed, 2003). O primeiro caso de Lolium multiflorum resistente ao glyphosate foi relatado por 
Perez \& Kogan (2002). O biótipo resistente foi identificado em pomares no Chile, que vinham recebendo, em média, três aplicações de glyphosate por ciclo durante os últimos 8 a 10 anos (Perez \& Kogan, 2002). Após 15 anos de uso bem sucedido de glyphosate na Austrália, foram identificados biótipos de Lolium rigidum resistentes a este herbicida (Powles et al., 1998). Os biótipos resistem a doses 7 a 11 vezes maiores de glyphosate do que os biótipos considerados sensiveis e apresentam-se suscetiveis a herbicidas com outros mecanismos de ação, como paraquat, sethoxydim, simazine e fluazifop (Powles et al., 1998). O objetivo deste trabalho foi avaliar a resposta de uma população de plantas de azevém, suspeita de resistência, ao herbicida glyphosate em pomar de maçã.

\section{MATERIAL E MÉTODOS}

Foram conduzidos três experimentos, sendo um em campo, em pomar de maçã em Vacaria-RS, e dois em casa de vegetação, na Estação Experimental de Fruticultura Temperada da Embrapa Uva e Vinho, na mesma cidade.

Os tratamentos herbicidas, tanto nos experimentos em casa de vegetação quanto em campo, foram aplicados com aspersor costal de precisão, com volume de calda de $150 \mathrm{~L} \mathrm{ha}^{-1}$. O delineamento experimental usado foi o completamente casualizado, com quatro repetições. A toxicidade dos tratamentos herbicidas foi avaliada utilizando-se escala percentual, em que a nota zero significou nenhum efeito de dano às plantas e nota 100 representou morte ou completa supressão destas. A toxicidade dos tratamentos herbicidas foi avaliada aos 15, 30 e 45 DAT (dias após tratamento).

No experimento realizado em campo, foram aplicadas doses crescentes do herbicida glyphosate $(0,360,720,1.440,2.880,5.760$ e 11.520 g e.a. ha ${ }^{-1}$, correspondentes a $0,1,2$, 4, 8, 16 e $32 \mathrm{~L} \mathrm{ha}^{-1}$, respectivamente, do produto comercial) mais os herbicidas paraquat (600 g i. a. ha-1), glufosinate (400 g i.a. ha-1), haloxyfop (120 g i.a. ha h $^{-1}$ e diclofop (426 g i.a. $\mathrm{ha}^{-1}$ ) em dois estádios vegetativos das plantas de azevém (três a quatro folhas e prefloração).

As plantas que sobreviveram à maior dose de glyphosate (11.520 g e.a. ha $\left.{ }^{-1}\right)$ aplicada no experimento em campo tiveram suas sementes colhidas e utilizadas nos experimentos em casa de vegetação, sendo aceitas como resistentes ao glyphosate. Para servir como propágulos de individuos representantes de uma população sensível ao produto, foram colhidas sementes de plantas que vegetavam em uma área da estação experimental da Embrapa Uva e Vinho, em Vacaria-RS, onde não se utiliza o herbicida glyphosate.

Para realização dos experimentos em casa de vegetação foram semeadas 10 sementes de azevém em recipientes com capacidade para $500 \mathrm{~mL}$. Após a emergência das plantas, procedeu-se ao desbaste, deixando quatro plantas por vaso. Essas plantas, ao atingirem estádio de três a quatro folhas, foram tratadas com doses crescentes do herbicida glyphosate $(0,360,720,1.440,2.880$ e 5.760 g e.a. ha ${ }^{-1}$ ) e com os herbicidas paraquat (600 g i.a. ha ${ }^{-1}$ ), glufosinate (400 g i. a. ha-1), haloxyfop (120 g i.a. ha ${ }^{-1}$ ) e diclofop-methyl (426 g i.a. ha ${ }^{-1}$ ), como tratamentos-padrão. As plantas que sobreviveram à maior dose do glyphosate (5.760 g e.a ha ${ }^{-1}$ ) foram mantidas em bulk (agrupadas) para produção de sementes e cruzamentos, para utilização em estudos posteriores.

No segundo experimento em casa de vegetação foram aplicados, em pós-emergência, no estádio de três a quatro folhas, os tratamentos herbicidas: glyphosate (720 g e.a. ha $\left.{ }^{-1}\right)$, glyphosate (1.440 g e.a. ha $\left.{ }^{-1}\right)$ : glyphosate (2.880 g e.a. ha ${ }^{-1}$ ), em seqüencial, glyphosate $\left(720+720\right.$ g e.a. ha $\left.{ }^{-1}\right)$, glyphosate $(720+$ 1.440 g e.a. ha-1), paraquat (600 g i.a. ha-1), glufosinate $\left(400 \mathrm{~g}\right.$ i.a. ha $\left.\mathrm{h}^{-1}\right)$, haloxyfop (120 g i.a. ha-1), clethodim (120 g i. a. ha-1), sethoxydim (184 g i.a. ha $\left.\mathrm{h}^{-1}\right)$, diclofop (426 g i.a. ha-1), fenoxaprop (110 g i.a. ha-1), fluazifop (250 g i.a. ha ${ }^{-1}$ ), paraquat + diuron (300 + 600 g i.a. ha ${ }^{-1}$ ), e, em pré-emergência: atrazine + simazine $(1.500+1.500$ g i.a. ha-1 $)$, trifluralin (3.000 g i.a. ha ${ }^{-1}$ ) e metolachlor (1.920 g i.a. ha ${ }^{-1}$ ), sobre vasos contendo o biótipo resistente e outros contendo o sensivel. A segunda dose dos tratamentos seqüenciais foi aplicada 15 dias após a primeira.

Os dados obtidos nos experimentos foram submetidos à análise de variância e, quando constatada significância, as médias foram comparadas entre si pelo teste de Duncan a $5 \%$ de probabilidade. 


\section{RESULTADOS E DISCUSSÃO}

No experimento em campo, observou-se que, aos 45 DAT, o glyphosate, nas doses de até $1.440 \mathrm{~g}$ e.a. ha $^{-1}$, produziu toxicidade inferior a $15 \%$ nos dois estádios vegetativos avaliados, enquanto em doses de até 5.760 g e.a. ha-1 ele produziu toxicidade máxima de $45 \%$ nestes estádios. Já a maior dose de glyphosate avaliada, 11.520 g e.a. ha ${ }^{-1}$, produziu toxicidade de $80 \%$ (Tabela 1). Os demais tratamentos herbicidas, com mecanismos de ação distintos daqueles do glyphosate, apresentaram altos níveis de controle, acima de $90 \%$, independentemente do estádio de aplicação, com exceção do glufosinate, que na aplicação em prefloração apresentou controle de apenas 75\%, considerado baixo, devido à alta capacidade competitiva e ao grande número de sementes produzidas por essa espécie.

Dessa forma, aproximadamente $85 \%$ das plantas da população não são controladas com doses de até 1.440 g e.a. ha ${ }^{-1}$ de glyphosate e aproximadamente $20 \%$ não respondem à dose de até 11.520 g e.a. ha ${ }^{-1}$, ou seja, não respondem à dose dezesseis vezes maior que a menor dose normalmente utilizada (720 g e.a. ha ${ }^{-1}$ de glyphosate) para controle dessa graminea. Esses resultados indicam que o glyphosate apresenta reduzida atividade sobre esta população de azevém e que herbicidas com outros mecanismos de ação apresentam controle elevado desta (Tabela 1). Resultados semelhantes foram obtidos por Perez \& Kogan (2002), quando biótipos de Lolium multiflorum, avaliados pelos autores, sobreviveram a doses de até 4.320 g e.a. ha-1 de glyphosate.

No primeiro experimento realizado em casa de vegetação, observou-se que a menor dose do herbicida glyphosate (360 g e.a. ha-1) foi suficiente para controlar totalmente o biótipo sensível já aos 15 DAT (Tabela 2). Já o biótipo resistente manifestou toxicidade de $45 \%$ para a maior dose de glyphosate avaliada (5.760 g e.a. ha-1), evidenciando, novamente, a baixa atividade da molécula neste biótipo. Os demais herbicidas avaliados, com mecanismos de ação distintos daqueles do glyphosate, apresentaram controle acima de $90 \%$ na avaliação realizada aos 15 DAT e de $100 \%$ na avaliação aos 30 DAT, tanto do biótipo sensivel quanto do resistente (Tabela 2).

No segundo experimento realizado em casa de vegetação, os resultados obtidos evidenciam, novamente, que a dose de $360 \mathrm{~g}$ e.a. $\mathrm{ha}^{-1}$ de glyphosate foi suficiente para controlar o biótipo sensivel (Tabela 3). O biótipo resistente manifestou, novamente, baixa toxicidade - inferior a 35\% - em resposta aos tratamentos com glyphosate, mesmo quando este foi aplicado de forma seqüencial. Não

Tabela 1 - Avaliação de toxicidade aos 15, 30 e 45 dias após o tratamento (DAT), provocada por tratamentos herbicidas aplicados sobre azevém (Lolium multiflorum) em campo em dois estádios vegetativos. Embrapa Uva e Vinho - VacariaRS, 2003

\begin{tabular}{|c|c|c|c|c|c|c|c|}
\hline \multirow{3}{*}{ Tratamento } & \multirow{3}{*}{$\begin{array}{c}\text { Dose } \\
\left(\mathrm{g} \mathrm{ha}^{-1}\right)\end{array}$} & \multicolumn{6}{|c|}{ Toxicidade $(\%)$} \\
\hline & & \multicolumn{3}{|c|}{ Estádio de aplicação 3-4 folhas } & \multicolumn{3}{|c|}{ Estádio de aplicação prefloração } \\
\hline & & $15 \mathrm{DAT}$ & $30 \mathrm{DAT}$ & 45 DAT & $15 \mathrm{DAT}$ & $30 \mathrm{DAT}$ & $45 \mathrm{DAT}$ \\
\hline Testemunha & --- & $0 \mathrm{f}^{*}$ & $0 \mathrm{e}$ & $0 \mathrm{f}$ & $0 \mathrm{f}$ & $0 \mathrm{f}$ & $0 \mathrm{f}$ \\
\hline Glyphosate & 360 e.a. & $0 \mathrm{f}$ & $0 \mathrm{e}$ & $0 \mathrm{f}$ & $0 \mathrm{f}$ & $0 \mathrm{f}$ & $0 \mathrm{f}$ \\
\hline Glyphosate & 720 e.a. & 6 ef & $14 \mathrm{~d}$ & $12 \mathrm{e}$ & $5 \mathrm{ef}$ & $5 \mathrm{ef}$ & 5 ef \\
\hline Glyphosate & 1.440 e.a. & $11 \mathrm{e}$ & $20 \mathrm{~d}$ & $15 \mathrm{e}$ & $12 \mathrm{de}$ & $10 \mathrm{de}$ & $10 \mathrm{de}$ \\
\hline Glyphosate & 2.880 e.a. & $25 \mathrm{~d}$ & $40 \mathrm{c}$ & $30 \mathrm{~d}$ & $18 \mathrm{~d}$ & $20 \mathrm{~cd}$ & $20 \mathrm{~d}$ \\
\hline Glyphosate & 5.760 e.a. & $38 \mathrm{c}$ & $48 c$ & $45 c$ & $35 \mathrm{c}$ & $30 c$ & $40 \mathrm{c}$ \\
\hline Glyphosate & 11.520 e.a. & $70 \mathrm{~b}$ & $76 \mathrm{~b}$ & $80 \mathrm{~b}$ & $75 \mathrm{~b}$ & $70 \mathrm{~b}$ & $75 \mathrm{~b}$ \\
\hline Paraquat & 600 i.a. & $100 \mathrm{a}$ & $100 \mathrm{a}$ & $100 a$ & $100 a$ & $100 \mathrm{a}$ & $100 \mathrm{a}$ \\
\hline Glufosinate & 400 i.a. & $75 \mathrm{~b}$ & $90 a$ & $100 \mathrm{a}$ & $70 \mathrm{~b}$ & $80 \mathrm{~b}$ & $75 \mathrm{~b}$ \\
\hline Haloxyfop & 120 i.a. & $90 \mathrm{a}$ & $100 \mathrm{a}$ & $100 \mathrm{a}$ & $92 \mathrm{a}$ & $95 \mathrm{a}$ & $95 \mathrm{a}$ \\
\hline Diclofop & 426 i.a. & $92 \mathrm{a}$ & $90 \mathrm{a}$ & $100 \mathrm{a}$ & $90 \mathrm{a}$ & $92 \mathrm{a}$ & $90 \mathrm{a}$ \\
\hline \multicolumn{2}{|c|}{ Coeficiente de variação } & 10,5 & 8,2 & 11,6 & 13,8 & 14,9 & 13,4 \\
\hline
\end{tabular}

* Médias seguidas pela mesma letra, na coluna, não diferem entre si pelo teste de Duncan a $5 \%$ de probabilidade. 
houve diferença significativa entre a aplicação de 1.440 g e.a. ha-1 de glyphosate em uma única vez e a aplicação seqüencial de $720+720$ g e.a. ha- ${ }^{-1}$ O aumento da dose utilizada em seqüencial $\left(720+1.440\right.$ g e.a. ha ${ }^{-1}$ de glyphosate) também não proporcionou incremento significativo no controle, não diferenciando, estatisticamente, da dose

Tabela 2 - Avaliação de toxicidade aos 15, 30 e 45 dias após o tratamento (DAT), provocada por doses crescentes de glyphosate e herbicidas com diferentes mecanismos de ação, aplicados sobre um biótipo de azevém (Lolium multiflorum) resistente e um sensível em casa de vegetação. Embrapa Uva e Vinho - Vacaria-RS, 2003

\begin{tabular}{|c|c|c|c|c|c|c|c|}
\hline \multirow{3}{*}{ Tratamento } & \multirow{3}{*}{$\begin{array}{l}\text { Dose } \\
\left(\mathrm{g} \mathrm{ha}^{-1}\right)\end{array}$} & \multicolumn{6}{|c|}{ Toxicidade (\%) } \\
\hline & & \multicolumn{3}{|c|}{ Sensível } & \multicolumn{3}{|c|}{ Resistente } \\
\hline & & $15 \mathrm{DAT}$ & $30 \mathrm{DAT}$ & 45 DAT & $15 \mathrm{DAT}$ & 30 DAT & $45 \mathrm{DAT}$ \\
\hline Testemunha & $\ldots$ & $0 \mathrm{~b}^{*}$ & $0 \mathrm{~b}$ & $0 \mathrm{~b}$ & $0 \mathrm{e}$ & $0 \mathrm{~d}$ & $0 \mathrm{e}$ \\
\hline Glyphosate & 360 e.a. & $100 \mathrm{a}$ & $100 \mathrm{a}$ & $100 \mathrm{a}$ & $0 \mathrm{e}$ & $0 \mathrm{~d}$ & $0 \mathrm{e}$ \\
\hline Glyphosate & 720 e.a. & $100 \mathrm{a}$ & $100 \mathrm{a}$ & $100 \mathrm{a}$ & $7 \mathrm{de}$ & $9 \mathrm{~cd}$ & $8 \mathrm{de}$ \\
\hline Glyphosate & 1.440 e.a. & $100 \mathrm{a}$ & $100 \mathrm{a}$ & $100 \mathrm{a}$ & $15 \mathrm{~cd}$ & $16 \mathrm{c}$ & $18 \mathrm{~cd}$ \\
\hline Glyphosate & 2.880 e.a. & $100 \mathrm{a}$ & $100 \mathrm{a}$ & $100 \mathrm{a}$ & $25 \mathrm{c}$ & $38 \mathrm{~b}$ & $25 \mathrm{c}$ \\
\hline Glyphosate & 5.760 e.a. & $100 \mathrm{a}$ & $100 \mathrm{a}$ & $100 \mathrm{a}$ & $40 \mathrm{~b}$ & $45 \mathrm{~b}$ & $45 \mathrm{~b}$ \\
\hline Paraquat & 600 i.a. & $100 \mathrm{a}$ & $100 \mathrm{a}$ & $100 \mathrm{a}$ & $100 \mathrm{a}$ & $100 \mathrm{a}$ & $100 \mathrm{a}$ \\
\hline Glufosinate & 400 i.a. & $100 \mathrm{a}$ & $100 \mathrm{a}$ & $100 \mathrm{a}$ & $96 \mathrm{a}$ & $100 \mathrm{a}$ & $100 \mathrm{a}$ \\
\hline Haloxyfop & 120 i.a. & $93 \mathrm{a}$ & $100 \mathrm{a}$ & $100 \mathrm{a}$ & $90 \mathrm{a}$ & $100 \mathrm{a}$ & $100 \mathrm{a}$ \\
\hline Diclofop & 426 i.a. & $95 \mathrm{a}$ & $100 \mathrm{a}$ & $100 \mathrm{a}$ & $92 \mathrm{a}$ & $100 \mathrm{a}$ & $100 \mathrm{a}$ \\
\hline \multicolumn{2}{|c|}{ Coeficiente de variação } & 8,2 & 9,5 & 8,6 & 10,3 & 11,7 & 11,1 \\
\hline
\end{tabular}

* Médias seguidas pela mesma letra, na coluna, não diferem entre si pelo teste de Duncan a $5 \%$ de probabilidade.

Tabela 3 - Avaliação de toxicidade aos 15, 30 e 45 dias após o tratamento (DAT), provocada por diferentes doses de glyphosate e herbicidas com diferentes mecanismos de ação, aplicados sobre um biótipo de azevém (Lolium multiflorum) resistente e um sensível em casa de vegetação. Embrapa Uva e Vinho - Vacaria-RS, 2003

\begin{tabular}{|c|c|c|c|c|c|c|c|}
\hline \multirow{3}{*}{ Tratamento } & \multirow{3}{*}{$\begin{array}{l}\text { Dose } \\
\left(\mathrm{g} \mathrm{ha}^{-1}\right)\end{array}$} & \multicolumn{6}{|c|}{ Toxicidade $(\%)$} \\
\hline & & \multicolumn{3}{|c|}{ Sensível } & \multicolumn{3}{|c|}{ Resistente } \\
\hline & & $15 \mathrm{DAT}$ & 30 DAT & 45 DAT & $15 \mathrm{DAT}$ & 30 DAT & 45 DAT \\
\hline Testemunha & $-\cdots$ & $0 \mathrm{c}^{*}$ & $0 \mathrm{~b}$ & $0 \mathrm{~b}$ & $0 \mathrm{e}$ & $0 \mathrm{~d}$ & $0 \mathrm{~d}$ \\
\hline Glyphosate & 720 e.a. & $100 \mathrm{a}$ & $100 \mathrm{a}$ & $100 \mathrm{a}$ & $5 \mathrm{e}$ & $6 \mathrm{~cd}$ & $10 \mathrm{c}$ \\
\hline Glyphosate & 1.440 e.a. & $100 \mathrm{a}$ & $100 \mathrm{a}$ & $100 \mathrm{a}$ & $8 \mathrm{e}$ & $10 \mathrm{c}$ & $12 \mathrm{c}$ \\
\hline Glyphosate & 2.880 e.a. & $100 \mathrm{a}$ & $100 \mathrm{a}$ & $100 \mathrm{a}$ & $20 \mathrm{~d}$ & $30 \mathrm{~b}$ & $30 \mathrm{~b}$ \\
\hline Glyphosate & $720+720$ e.a. & $100 \mathrm{a}$ & $100 \mathrm{a}$ & $100 \mathrm{a}$ & $8 \mathrm{e}$ & $15 \mathrm{c}$ & 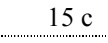 \\
\hline Glyphosate & $720+1440$ e.a. & $100 \mathrm{a}$ & $100 \mathrm{a}$ & $100 \mathrm{a}$ & $10 \mathrm{e}$ & $35 \mathrm{~b}$ & $35 \mathrm{~b}$ \\
\hline Paraquat & 600 i.a. & $100 \mathrm{a}$ & $100 \mathrm{a}$ & $100 \mathrm{a}$ & $100 \mathrm{a}$ & $100 \mathrm{a}$ & $100 \mathrm{a}$ \\
\hline Glufosinate & 400 i.a. & $100 \mathrm{a}$ & $100 \mathrm{a}$ & $100 \mathrm{a}$ & $91 \mathrm{a}$ & $100 \mathrm{a}$ & $100 \mathrm{a}$ \\
\hline Haloxyfop & 120 i.a. & $100 \mathrm{a}$ & $100 \mathrm{a}$ & $100 \mathrm{a}$ & $90 \mathrm{a}$ & $100 \mathrm{a}$ & $100 \mathrm{a}$ \\
\hline Clethodim & 120 i.a. & $100 \mathrm{a}$ & $100 \mathrm{a}$ & $100 \mathrm{a}$ & $98 \mathrm{a}$ & $100 \mathrm{a}$ & $100 \mathrm{a}$ \\
\hline Sethoxydim & 184 i.a. & $100 \mathrm{a}$ & $100 \mathrm{a}$ & $100 \mathrm{a}$ & $95 \mathrm{a}$ & $100 \mathrm{a}$ & $\begin{array}{l}100 \mathrm{a} \\
100 \mathrm{a} \\
\end{array}$ \\
\hline Diclofop & 426 i.a. & $100 \mathrm{a}$ & $100 \mathrm{a}$ & $100 \mathrm{a}$ & $92 \mathrm{a}$ & $100 \mathrm{a}$ & $100 \mathrm{a}$ \\
\hline Fenoxaprop & 110 i.a. & $100 \mathrm{a}$ & $100 \mathrm{a}$ & $100 \mathrm{a}$ & $90 \mathrm{a}$ & $100 \mathrm{a}$ & $100 \mathrm{a}$ \\
\hline Fluazifop & 250 i.a. & $100 \mathrm{a}$ & $100 \mathrm{a}$ & $100 \mathrm{a}$ & $95 \mathrm{a}$ & $100 \mathrm{a}$ & $100 \mathrm{a}$ \\
\hline Paraquat + diuron & $300+600$ i.a. & $100 \mathrm{a}$ & $100 \mathrm{a}$ & $100 \mathrm{a}$ & $100 \mathrm{a}$ & $100 \mathrm{a}$ & $100 \mathrm{a}$ \\
\hline Atrazine + simazine & $1.500+1.500$ i.a. & $15 \mathrm{~b}$ & $100 \mathrm{a}$ & $100 \mathrm{a}$ & $10 \mathrm{e}$ & $100 \mathrm{a}$ & $100 \mathrm{a}$ \\
\hline Trifluralin & 3.000 i.a. & $100 \mathrm{a}$ & $100 \mathrm{a}$ & $100 \mathrm{a}$ & $100 \mathrm{a}$ & $100 \mathrm{a}$ & $100 \mathrm{a}$ \\
\hline Metolachlor & 1.920 i.a. & $100 \mathrm{a}$ & $100 \mathrm{a}$ & $100 \mathrm{a}$ & $100 \mathrm{a}$ & $100 \mathrm{a}$ & $100 \mathrm{a}$ \\
\hline \multicolumn{2}{|c|}{ Coeficiente de variação } & 7,5 & 5,4 & 5,6 & 10,9 & 11,2 & 11,4 \\
\hline
\end{tabular}

* Médias seguidas pela mesma letra, na coluna, não diferem entre si pelo teste de Duncan a 5\% de probabilidade. 
de 2.880 g e.a. ha-1 de glyphosate (Tabela 3). Assim, a aplicação seqüencial não proporcionou aumento na atividade do glyphosate sobre o biótipo resistente, evidenciando não ser uma alternativa viável. Já os herbicidas paraquat, glufosinate, haloxyfop, clethodim, sethoxydim, diclofop, fenoxaprop, fluazifop, paraquat + diuron, atrazine + simazine, trifluralin e metolachlor controlaram com eficiência os biótipos sensivel e resistente (Tabela 3), apresentando-se como possiveis produtos alternativos para uso em áreas infestadas com o biótipo resistente. Em trabalho semelhante, Powles et al. (1998) obtiveram controle de Lolium rigidum resistente ao glyphosate com os herbicidas paraquat, sethoxydim, simazine e fluazifop.

Os resultados obtidos nos experimentos em campo e em casa de vegetação, de forma geral, evidenciam que o biótipo suscetivel é facilmente controlado com o herbicida glyphosate e pelos demais herbicidas pósemergentes avaliados, independentemente do estádio vegetativo. Além disso, demonstram que o biótipo resistente apresenta-se, igualmente ao biótipo sensivel, altamente suscetível aos herbicidas com mecanismo de ação distinto do glyphosate, independentemente do estádio vegetativo. Entretanto, o biótipo resistente apresenta baixa resposta ao herbicida glyphosate, mesmo se este for empregado de forma seqüencial e/ou em altas doses, evidenciando ter adquirido resistência a este produto.

Os resultados permitem concluir que: aproximadamente $80 \%$ das plantas da população avaliada resistem a doses de até $1.440 \mathrm{~g}$ e.a. ha ${ }^{-1}$ de glyphosate e, aproximadamente, $20 \%$ a doses de até 11.520 g e.a. ha ${ }^{-1}$; aplicações seqüenciais não aumentam a atividade do glyphosate sobre o biótipo resistente; a dose de 360 g e. a. ha ${ }^{-1}$ de glyphosate é suficiente para controlar o biótipo sensivel, independentemente do estádio vegetativo; os herbicidas paraquat, glufosinate, haloxyfop, clethodim, sethoxydim, diclofop, fenoxaprop, fluazifop, paraquat + diuron, atrazine + simazine, trifluralin e metolachlor controlam com eficiência os biótipos sensível e resistente; e a sensibilidade do biótipo resistente a herbicidas com mecanismos de ação distintos dos do glyphosate descarta a possibilidade de resistência múltipla aos produtos avaliados.

\section{LITERATURA CITADA}

BETTS, K. J. et al. Mechanism of inheritance of diclofop resistance in italian ryegrass (Lolium multiflorum). Weed Sci., v. 40, n. 2, p. 184-189, 1992.

HOLT, J. S.; LEBARON, H. M. Significance and distribution of herbicide resistance. Weed Technol., v. 4, n. 1, p. 141-149, 1990 .

PEREZ, A.; KOGAN, M. Glyphosate-resistant Lolium multiflorum in Chilean orchards. Weed Research, v. 43, p. 12-19, 2002.

POWLES, S. B.; HOLTUM, J. A. M. Herbicide resistance in plants: biology and biochemistry. Boca Raton: 1994. 353 p.

POWLES, S. B. et al. Envolved resistance to glyphosate in rigid ryegrass (Lolium rigidum) in Australia. Weed Sci., v. 46 , p. $604-607,1998$

WEED SCIENCE. Official WSSA definitions. Disponível em: <http://www.weedscience.com/paper/ definitions.htm>. Acesso em: 22 abr. 1999.

WEED SCIENCE - Glycines (g/9) resistant weeds by species and country. Disponível em: $<\mathrm{http}: / /$ www.weedscience.org/Summary/ UspeciesMOA.asp?1stMOAID $=12 \&$ FmHRACGroup $=$ Go $>$. Acesso em: 10 fev. 2003. 